\title{
Plastic Instability in Omega Forming Alloy Systems
}

\section{S.L. Wadekar}

Materials Science Division

Bhabha Atomic Research Centre

Mumbai 400 085, India

E-mail: wadekar@barc.ernet.in

\section{J.K. Chakravartty and S. Banerjee*}

Materials Science Division

*Materials Group

Bhabha Atomic Research Centre

Mumbai 400 085, India

E-mail: jayanta@apsara.barc.ernet.in,sbanerji@magnum.barc.ernet.in

\begin{abstract}
The plastic flow behaviour of two $\omega$ forming systems namely ordered Nb-40Ti-15Al and metastable $\mathrm{Zr}-\mathrm{Nb}$ alloys has been studied over a range of strain rate and temperature to establish the domain of serrated flow or Protevin-Le Chatelier (PLC) effect. The observed features of serrated flow in these alloys are strikingly similar to that observed during PLC effect of solid solution alloys. Samples deformed in the serrated flow regime have been studied at different levels of magnification using light, scanning electron and transmission electron microscopy. A very characteristic feature of deformation in these alloys has been the presence precipitate free channels, microbands and deformation bands. The proposed mechanism of serrated flow involves creation of soft channels formed by shearing of $\omega$-particles and dynamic restoration of $\omega$-particles within the soft channel resulting in pinning of dislocation. It appears that this is the characteristic feature of deformation of $\omega$ forming system exhibiting PLC effect.
\end{abstract}

International Conference on Statistical Mechanics

of Plasticity and Related Instabilities

Indian Institute of Science, Bangalore

August 29 - September 2, 2005

\section{${ }^{*}$ Speaker}




\section{Introduction}

A strong plastic instability leading to strain localization in the form of bands was reported by the authors in a omega $(\omega)$ forming body centered cubic (bcc) Ti-15Mo alloy at some combinations of temperature and strain rate during tensile deformation [1]. This instability manifested in the form of serrated flow was found to be similar to that observed during Protevin-Le Chatelier (PLC) effect in solid solution alloys. However, rather than attributing the serrated flow behaviour in Ti-15Mo with solute-dislocation interactions, they were linked with microscopic instability involved in the formation of $\omega$ phase [1]. Subsequently preliminary studies on ordered $\mathrm{Nb}$-Ti-Al alloys, in which $\omega$-phase forms during ageing revealed similar discontinuous yielding phenomena (serrated flow) and deformation characteristics [2]. From these studies, it appears that the formation and dissolution of $\omega$-phase dynamically during deformation may be responsible for the observed plastic instability and may be a characteristic feature of deformation in $\omega$-forming alloy systems.

It is well known that serrated flow or PLC effect in solid solution alloys is an extreme form of dynamic strain ageing (DSA) which results from the dynamic interaction between solute atoms and dislocations. A few of the commonly observed macroscopic manifestations of PLC effect are a discontinuous flow behaviour during plastic deformation, a negative strain rate sensitivity of stress (SRS), presence of critical strain for the initiation of serrations, and lowering of ductility in the serrated flow regime [3].

In view of the remarkable similarity in the manifestation of the plastic instability due to solute dislocation interaction, as in PLC effect, and those that are observed in the above mentioned omega forming systems, it is useful to study the nature of the instabilities in systems exhibiting varying degrees of stability with respect to omega formation. An additional insight into the mechanism can be provided, for example, by studying the influence of the kinetics of $\omega$ formation and stability of $\beta$ phase on the characteristics of serrated flow behaviour.

In this study, two omega forming systems $\mathrm{Nb}-\mathrm{Ti}-\mathrm{Al}$ and $\mathrm{Zr}-\mathrm{Nb}$ (with different $\mathrm{Nb}$ content) alloys have been chosen. The alloys of zirconium containing different levels of $\mathrm{Nb}$ have been chosen so as to modify the $\omega$-forming kinetics as well as to obtain $\beta$ phase with varying volume fraction [4]. These alloys were deformed at various temperature and strain rates to identify the domain of serrated flow and to study the manifestations of plastic instability. The deatails of the microstructural features of deformation have been charaterized using optical, scanning and transmission electron microscopes. An attempt has been made to rationalize the experimental observations using a model proposed earlier [5].

\section{Experimental}

Two $\omega$-forming alloy systems $\mathrm{Nb}-40 \mathrm{Ti}-15 \mathrm{Al}$ and $\mathrm{Zr}-\mathrm{Nb}$ alloys containing 12, 15 and 20 wt $\% \mathrm{Nb}$ were selected for this study. $\mathrm{Nb}-40 \mathrm{Ti}-15 \mathrm{Al}$ alloy was recrystallized at $1200^{\circ} \mathrm{C}$ and $\mathrm{Zr}$ $\mathrm{Nb}$ alloys were solutionized at $920^{\circ} \mathrm{C}$ for $20 \mathrm{~min}$. Subsequently these alloys were quenched in water. These $\beta$ quenched alloys of zircinium represent examples of metastable ( $\mathrm{Zr}-12 \mathrm{Nb}, \mathrm{Zr}$ - 
$15 \mathrm{Nb}$ ) and stable $(\mathrm{Zr}-20 \mathrm{Nb}) \beta$ alloys. Since $\mathrm{Nb}$ acts as $\beta$ stabilizer in $\mathrm{Zr}$, it can supress or modify the kinetics of of the formation of $\beta$ decomposion products such as $\alpha$ and both athermal and thermal $\omega$ phase. Tensile tests were carried out on specimens of $12.5 \mathrm{~mm}$ gauge length, 4 $\mathrm{mm}$ nominal width and $0.8 \mathrm{~mm}$ thickness. Uniaxial tensile tests upto fracture, and strain rate change tests were carried out at various cross-head speeds $(0.0005 \mathrm{~mm} / \mathrm{min}$ to $0.5 \mathrm{~mm} / \mathrm{min})$ and over a range of temperatures from 300 to $775 \mathrm{~K}$. Optical, scanning electron and transmission electron microscopes were used to characterize both microstructure and features of deformation.

\section{Results}

\subsection{Nb-Ti-Al Alloy}

TEM investigations revealed presence of ordered domains of the B2 structure (Fig.1(a)). Selected area diffraction (SAD) patterns from the B2 phase showed the presence of weak diffuse scattering along the $<112>$ direction of the B2 structure (Fig.1(b)-(d)). Such a pattern of diffuse scattering could be related to the presence of $\omega$ in the lattice (Fig.1). Tensile deformation carried out over a range of temperatures and strain rates exhibited two different types of plastic instabilities as per classification [6].

1. Clear yield point effect followed by work softening on deformation at room temperature as well as upon reloading subsequent to deformation and ageing at intermediate temperature (type-h instability, related to work softening) (see Fig.2 of [2]).

2. PLC effect or serrated flow behaviour over a range of temperature and strain rate (type-s instability related to negative strain rate sensitivity) (see Fig.4 of [2]).
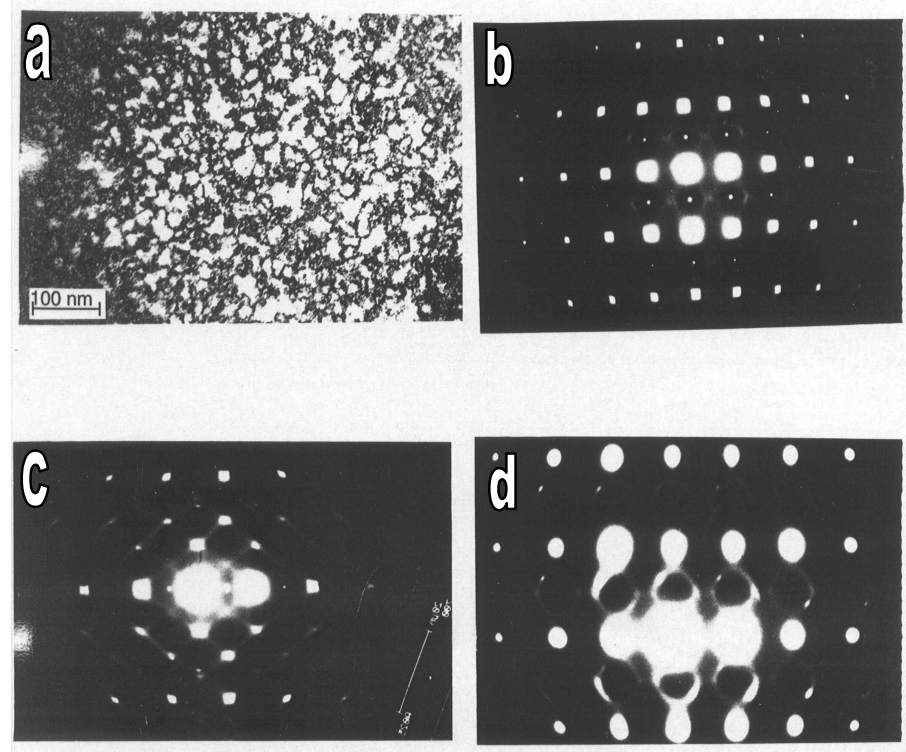

Fig.1 (a) Recrystallized microstructure of Nb-40Ti-15Al alloy, (b), (c) and (d) selected area diffraction patterns revealing presence of ordered B2 phase and of $\omega$. 
The range of strain rate and temperature over which serrated flow occurred was identified and the temperature range matched with the temperature range of formation of $\omega$ phase [2]. The PLC effect was accompanied by a negative strain rate sensitivity and occurred after some critical strain. A typical microstructure showing precipitate free soft channels created by shearing of $\omega$ particles could be clearly noticed in Fig. 2 and these were responsible for lack of work hardening. The dislocation arrangement in general was planar and the presence of dislocations pairs was noticed. Similar dislocation arrangements and patterns were also seen in the serrated flow regime of this material. In addition to these, localized slip along a crystallographic slip system lead to formation of large steps at grain boundaries (Fig.3).

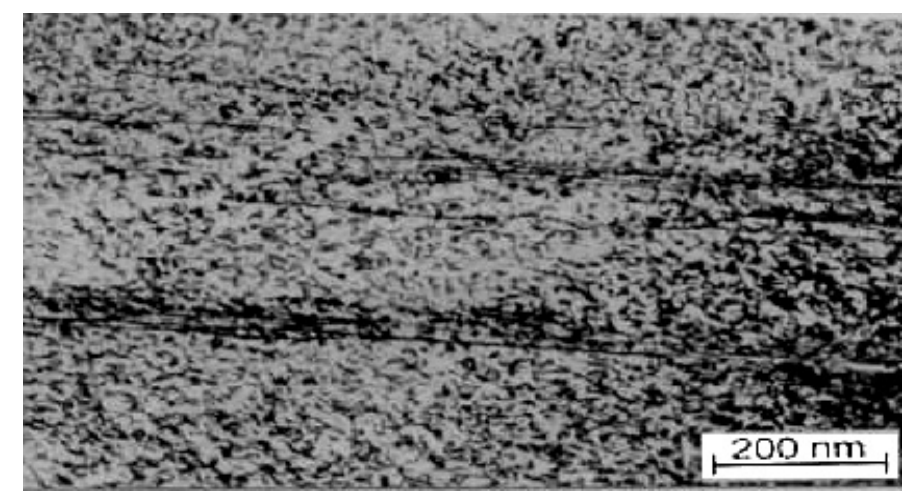

Fig.2 Formation of soft channels due to shearing of the $\omega$-particles in Ni-40Ti-15Al under deformed condition.

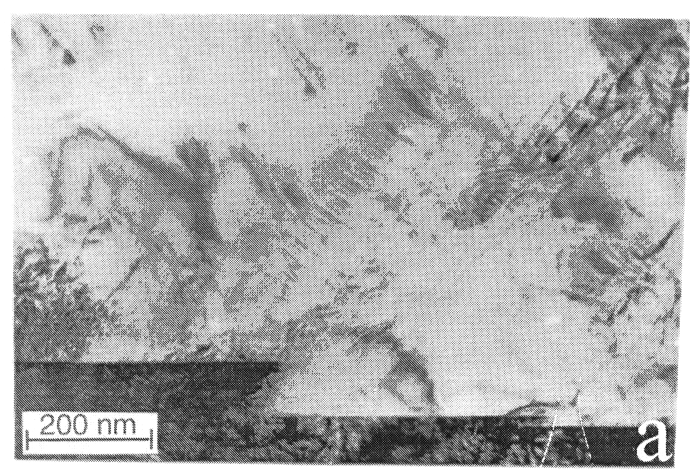

Fig.3: Planar array of dislocations and formation of large steps at grain boundaries in $\mathrm{Nb}-40 \mathrm{Ti}-15 \mathrm{Al}$ alloy during deformation in the serrated flow regime.

\subsection{Zr-Nb Alloy system}

$\mathrm{Zr}-12 \% \mathrm{Nb}$ and $\mathrm{Zr}-15 \% \mathrm{Nb}$ alloys exhibited fine $\omega$-particles distributed in equiaxed $\beta$ grains upon quenching from the single $\beta$ phase field. The $\mathrm{Zr}-12 \mathrm{Nb}$ alloy showed higher volume fraction of the $\omega$ phase as compared to the $\mathrm{Zr}-15 \mathrm{Nb}$ alloy. The $\mathrm{Zr}-20 \% \mathrm{Nb}$ alloy showed stable $\beta$ phase with no formation of $\omega$ on ageing. It has been seen that while the alloys with different starting microstructures showed homogeneous flow behaviour at room temperature, a 
considerable variation in the flow characteristics has been noted during deformation at elevated temperatures (Fig.4). Following are some of the important observations made during tensile deformation.

1. The $\mathrm{Zr}-20 \% \mathrm{Nb}$ alloy which does not form athermal $\omega$-phase showed homogeneous flow behaviour at all deformation temperatures employed as revealed by smooth loaddisplacement plots (Fig. 4).

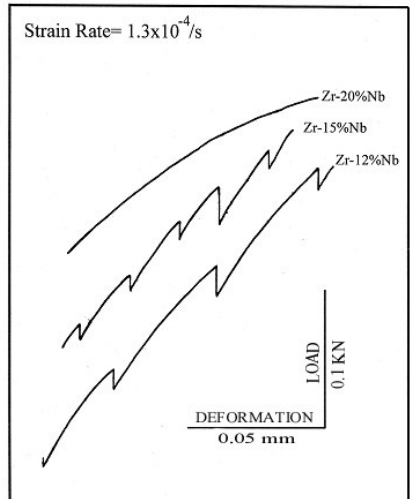

Fig.4 Load-displacement behaviour of different $\mathrm{Zr}-\mathrm{Nb}$ alloys at $623 \mathrm{~K}$

2. $\mathrm{Zr}-12 \%$ and $\mathrm{Zr}-15 \% \mathrm{Nb}$ alloys exhibit plastic instability in the form of serrated flow behaviour at some combinations of strain rate and temperature (Fig.4). A typical domain of serrated flow in temperature-strain rate plot for the $\mathrm{Zr}-15 \% \mathrm{Nb}$ alloy is shown in Fig.5. It may be noted that while the strain rate for the appearance of serration is temperature dependent, the strain rate for disappearnace of serration is athermal.

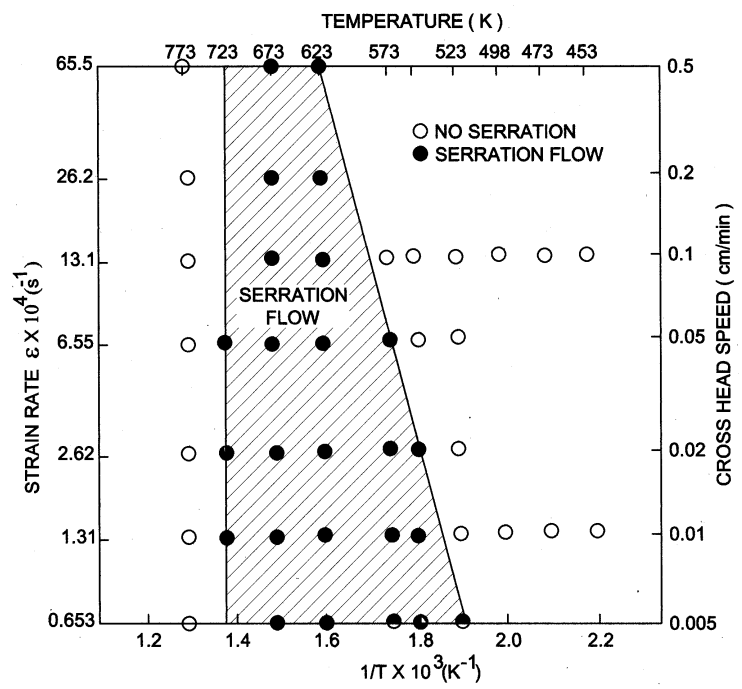

Fig. 5: Domain of temperature and strain rate for serrated flow in $\mathrm{Zr}-15 \mathrm{Nb}$ alloy. 


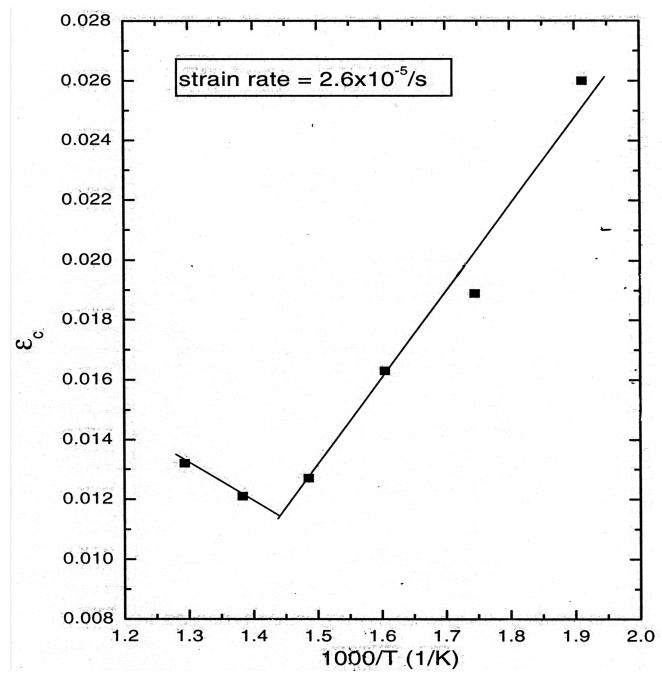

Fig.6 Critical strain $\left(\varepsilon_{\mathrm{c}}\right)$ as a function of temperature in $\mathrm{Zr}-15 \mathrm{Nb}$ alloy.

3. A critical strain is always involved with the onset of serrated flow and the extent of critical strain is observed to be a function of strain rate and temperature (Fig. 6). It is seen that at lower temperatures critical strain decreases with increasing temperature, referred to as a normal behaviour. However, a change over from normal to inverse behaviour is noted at around $673 \mathrm{~K}$ for $\mathrm{Zr}-15 \mathrm{Nb}$ alloy. Further, under similar deformation condition, the magnitude of the onset strain $\left(\varepsilon_{\mathrm{c}}\right)$ for serrated flow appears to be lower in $\mathrm{Zr}-12 \mathrm{Nb}$.

4. A negative strain rate sensitivity of stress is recorded in the serrated flow regime (Fig.7).

5. The serrations are primarily of C-type. The stress drop associated with serrated flow is found to be dependent on strain rate and temperature for a given alloy. For a given strain rate and temperature the stress drops are larger in $\mathrm{Zr}-12 \mathrm{Nb}$ as compared to $\mathrm{Zr}-15 \mathrm{Nb}$. However strain-time delay between the two successive serrations shows irregular variations with strain rate, temperature and $\mathrm{Nb}$ content.

Deformed microstructures (in the serrated flow regime) observed in SEM showed the presence of well developed and uniformly distributed slip lines (Fig.8). These lines are essentially straight during the initial stages of deformation. With increasing strain, the step like slip pattern results presumably from the intersection of activated secondary slip lines. These slip lines are found to be confined within the $\beta$ grains as shown by arrow in Fig 8. Fig.9 shows TEM micrographs of deformed sample of $\mathrm{Zr}-15 \mathrm{Nb}$ revealing the presence of planar array of single dislocations and microbands which appear to be aligned along some crystallographic slip planes. The microbands are essentially plate like channels $100 \mathrm{~nm}$ thick within which intense dislocation activity results from the localized flow. The dislocation density within the bands appears to vary, as both high dislocation density islands and almost dislocation free areas, have been seen (Fig.10). In addition to these, diffraction contrast experiments have revealed presence of fine distribution of $\omega$-precipitates within the micro bands as well as in the matrix (Fig.11). Similar channels devoid of $\omega$-precipitates have also been observed inTi-15Mo alloy [1]. 


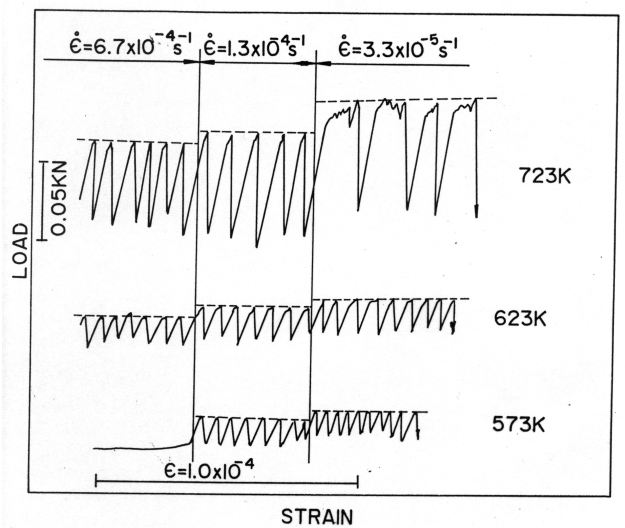

Fig.7 Increase in flow stress on reduction of strain rate (negative strain rate sensitivity) in $\mathrm{Zr}-15 \mathrm{Nb}$ alloy at various temperatures.

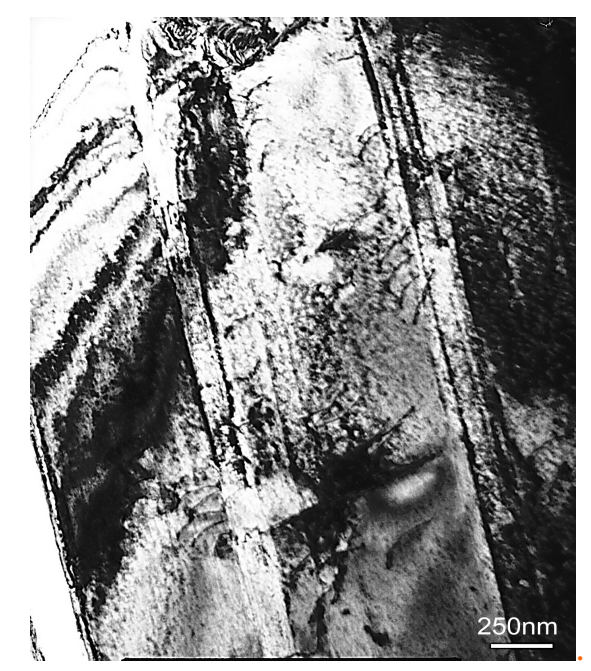

Fig. 9 Planar array of dislocations in Zr$15 \mathrm{Nb}$ alloy on deformation at $623 \mathrm{~K}$ and at strain rate of $1.3 \times 10^{-4} / \mathrm{s}$

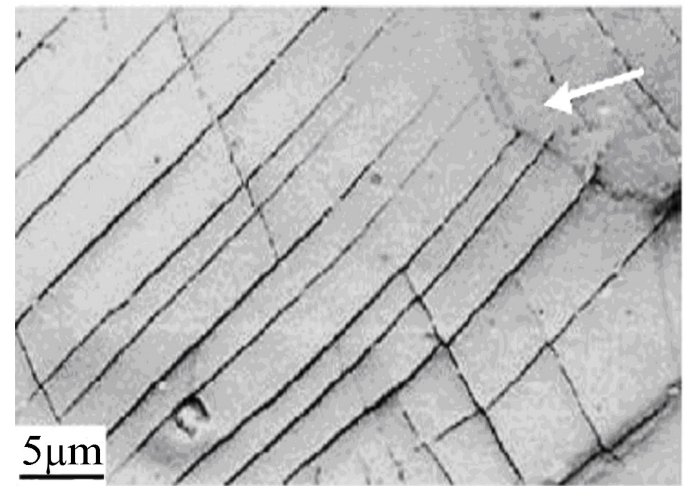

Fig. 8 Features of fine deformation bands in $\mathrm{Zr}-15 \mathrm{Nb}$ revealed with Scanning Electron Microscopy on deformation at $623 \mathrm{~K}$ with strain rate $1.3 \times 10^{-4} / \mathrm{s}$

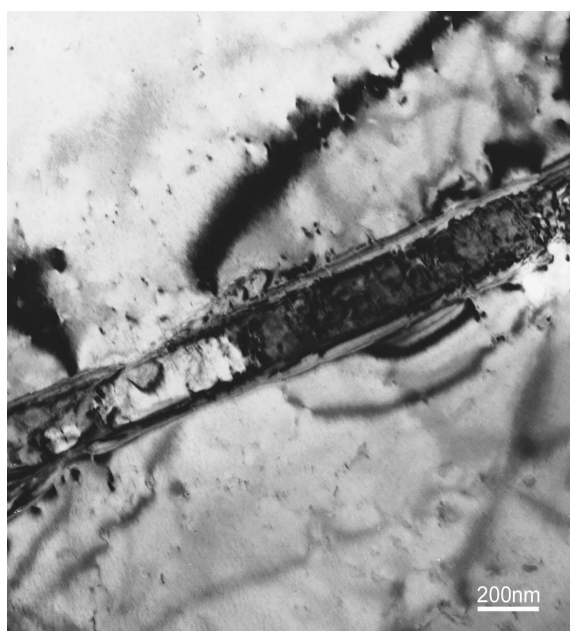

Fig. 10 Presence of $\omega$-precipitates within the microband as well as in the matrix in $\mathrm{Zr}-15 \mathrm{Nb}$ during deformation within serrated flow regime

The difference in contrast of $\omega$-precipitates, formed within band during cooling subsequent to deformation, from that within the matrix suggests that the orientation of $\beta$-region within the band is different from that of matrix $\beta$-grain. It is also noted that the crystallographically oriented microbands are not able to penetrate boundaries to propagate in to neighboring grain (Fig.12), but are found to create offsets in grain boundaries leading to strain concentration similar to that observed in Nb-40Ti-15Al (Fig.3). 


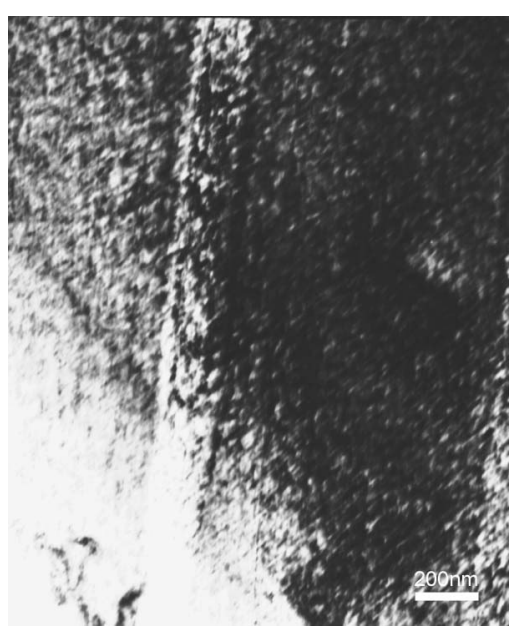

Fig.11 Micro-band formation due to shearing of the $\omega$-particles after deformation at $623 \mathrm{~K}$

\section{Discussion}

This investigation found that $\omega$ forming metastable $\beta$ alloys $\mathrm{Zr}-12 \mathrm{Nb}, \mathrm{Zr}-15 \mathrm{Nb}$, and ordered $\mathrm{Nb}-40 \mathrm{Ti}-15 \mathrm{Al}$ showed serrated flow behavior and negative strain rate sensitivity of stress in a particular strain rate and temperature domain. The serrations occurred after a finite critical strain, the magnitude of which was dependent on both temperature and strain rate. These observations are indicative of the fact that the three alloys studied are exhibiting PLC effect and that the observed variations of mechanical properties are similar to that have been reported in conventional PLC effect of solid solution alloys [3]. The trend of the present results is consistent with that reported earlier on Ti-Mo alloys [1]. Regarding the mechanism of serrated flow in these systems the following possibilities may be considered: (i) Cottrell's solute drag model in which an atmosphere is formed during dislocation movement [7], (ii) McCormick's [8] and Van den Beukel's [9] models on the basis of Sleeswyk's proposition [10] of solute segregation to dislocations during their arrest at obstacles, (iii) Rose and Glover's "pair reorientation model" based on Snoek theory in which dislocation movement is postulated to be hindered by interaction between the strain field of dislocation and that of vacancy-solute atom pairs [11] and (iv) Onodera et al's suggestion in which locking of dislocations is considered to be effected not by individual solute atoms but by clusters of solute atoms [12]. Although these models can be used to explain the various features of PLC effect observed in this investigation, the following observations appear to disqualify the aforementioned mechanisms which exclusively consider locking and unlocking of a dislocation by solute atoms.

a) The upper tempertaure limit of strain rate-temperature regime for metastable $\mathrm{Zr}-\mathrm{Nb}$ alloys is athermal which is not expected in solute pinning mechanisms.

b) The stable $\mathrm{Zr}-20 \mathrm{Nb}$ do not exhibit serrated flow at all strain rate-temperature combinations of this investigation and c) the observed variations of stress drop and critical strain in two metastable alloys of $\mathrm{Zr}$ (relatively larger stress drop and lower critical strain in $\mathrm{Zr}-12 \mathrm{Nb}$ alloy compared to $\mathrm{Zr}-15 \mathrm{Nb}$ alloy) are in contradiction with conventional PLC effect where increasing solute content enhances both critical strain and stress drop. 
Thus it appears that the mechanism of plastic instabilty in the $\omega$-forming system is complex and is certainly different from the simple picture of pinning of individual dislocations and their mobilization. It is known that simultaneous activation of large number of dislocations is required to cause a plastic instability during seratted flow. The in-situ high voltage TEM investigation of PLC effect in Al-Mg alloy [13] showed clearly the involvement of many dislocations during waiting at obstacles and in overcoming obstacles abruptly and collectively. As a consequence of these, the occurrence of the PLC effect has been treated on the basis of collective movement of a group of dislocations by Korbel [14] and Schoeck [15], and of propagation of localized deformation bands by Schlipf [16]. The latter models may be useful in understanding the plastic instability during serrated flow in $\omega$ forming systems. The TEM observation of deformed samples indicates a very inhomogeneous deformation mechanism where dislocations are restricted within the microbands or dislocation free channels. This localization of slip is promoted by by the shearing of $\omega$ particles and their mechanical dissolution. The mechanism of formation of the first PLC band (initiation of serration) can be considered to involve the following steps.

(a) Nucleation of microband, which corresponds to creation of a first avalanche of dislocations by shearing of pre-existing $\omega$-particles (Fig. 2), and subsequent grouping of these micro bands to form deformation bands within a $\beta$-grain (Fig. 8).

(b) As the deformation proceeds, deformation bands form in adjacent grains driven by stress concentration ahead of bands and requirement of strain compatibilty across the grain boundaries within the deforming volume of the sample. A PLC band comprising of these deformation bands ( $\omega$ free channels) triggers the first stress drop.

There is however a tendency for $\omega$ to re-precipitate depending on the chemical driving force for omega precipitation and elastic interaction between the strain fields associated with dislocation and the favorably oriented omega particles [5]. In this manner the successive operation of shearing, dissolution and re-precipitation of omega precipitates within the deformation channels lead to the serrated flow behaviour. It has been shown by Brechet and Estrin[17] that under certain conditions of deformation shearing of precipiates and their consequent dissolution may result in negative apparent strain rate senstitivity leading to plastic instabilty even in systems which do not exhibit conventional DSA. This phenomenon has been referred to as 'pseudo-PLC effect' and apparently resembles steps involved in PLC band formation in $\omega$ forming syatems. The present results complement the earlier works carried out in $\omega$ forming alloy systems in terms of the structural similarity in the respective plastic instability regimes [1,5]. A similar reasoning in the form of a model have been proposed to rationalize the observed features of deformation during PLC effect in Ti-15Mo alloy [5]. The same model may be invoked to establish the fact that serrated flow in the two different alloys of this study stems from the interaction of the $\omega$ precipitates with dislocations. This conclusion is drawn of the basis of three important observations of this study namely athermal nature of the upper temperature limit of strain rate-temperature for serrated flow, occurrence of serrated flow 
only in the temperature regime where $\omega$ phase can form, and reduced intensity of serrated flow in a solute rich alloy ( $\mathrm{Zr}-15 \mathrm{Nb}$ alloy vs. $\mathrm{Zr}-12 \mathrm{Nb}$ alloy).

\section{Conclusions:}

On the basis of results of this study the following general conclusion may be drawn : The plastic instability associated with serrated flow behaviour in Nb-40Ti-15Al, Zr-12Nb and Zr$15 \mathrm{Nb}$ alloys results from the interaction of $\omega$ particles with dislocations under temperature and strain-rate conditions favorable for dynamic formation of $\omega$-particles. The proposed mechanism of serrated flow involves creation of soft channels formed by shearing of $\omega$-particles and dynamic restoration of $\omega$-particles within the soft channel resulting in pinning of dislocation. It apperas that this is the characteristic feature of deformation of $\omega$ forming system exhibiting PLC effect.

\section{Acknowledgements:}

The authors are greatful to Dr. Rajeev Kapoor, Mr. C. Gupta and Dr. R. Tiwari for many useful discussions and for prepation of the manuscript.

\section{References}

[1] S. Banerjee, U. Naik, J.K. Chakravartty, Microscopic and macroscopic instabilities in omega forming systems, Key Eng. Mater., 103 (1995) 267-276.

[2] R.J. Grylls, S. Banerjee, S. Perungulam, R. Wheeler and H.L. Fraser, On the discontinuous yielding phenomena observed in a Nb-Ti_Al alloys, Intermetallics, 6 (1998) 749-752.

[3] P. Rodriguez, Serrated plstic flow, Bull. Mater. Sci., 6, 4 (1984) 653-663.

[4] R.F. Hehemann, Transactions in Zr-Nb alloy, Can. Met., Quart., 11 (1972) 201-212.

[5] S. Banerjee and U.M. Naik,Plastic instability in an omega forming Ti-15\%Mo alloy, Acta Mater., 44 (1996) 3667-3677.

[6] Y. Estrin and L.P. Kubin, Continuum models for materials with microstructure, edited by H.B. Muhlaus, (1995) 395-450.

[7] A.H. Cottrell, Effect of solute atoms on the behaviour of dislocations, Proc. Conf.on Strenghth of Solids, Bristol, (1947) 30-38.

[8] P.G. McCormick, A model for the Portevin-Li Chatelier effect in substitutional alloys, Acta Metal., 20 (1972) 351-354.

[9] A.van den Beukel, Theory of the effect of dynamic strain aging on mechanical properties, Phys. Stat. Sol., (A) 30 (1975) 197-206.

[10] A.W. Sleeswyk, Slow strain-rate hardening of ingot iron. Acta Metal., 6 (1958) 598-603.

[11] K.S.B. Rose and S.G. Glover, A study od strain ageing in austenite, Acta Metal., 14 (1966) 1505-1516.

[12] R. Onodera, H. Era, T. Ishabashi and M. Shimizu, Acta Metal., 31 (1983) 1589.

[13] T. Tabata, H. Fujita and Y. Nakajima, Behavior of dislocations in Al-Mg single crystals observed by high voltage elctron microscopy, Acta Metal, 28 (1980) 795-805.

[14] A. Korbel, J. Zasadzinski and Z. Sieklucka, A new approach to the Portevin-Lechatelier effect, Acta Metal., 24 (19976) 919-923.

[15] G. Schoeck, The Portevin-Le Chatelier effect a kinetic theory, Acta Metal., 32, 8 (1984) 12291234.

[16] J. Schlipf, Dislocation dynamics in strain aging alloys, Acta Metall. Mater., 40, 9 (1992) 20752084.

[17] Y. Brechet and Y. Estrin, On A Pseudo-Portevin -Le Chatelier Effect, Scripta. Metall. et Materialia, 31,2 (1994),185-190. 